\title{
Screening and Health Behaviors among Persons Diagnosed with Familial Adenomatous Polyposis and Their Relatives
}

\author{
Aimee S. James, ${ }^{1}$ Phillip Chisholm, ${ }^{2}$ Kathleen Y. Wolin, ${ }^{1}$ Melanie Baxter, ${ }^{3}$ \\ Kimberly Kaphingst, ${ }^{1}$ and Nicholas O. Davidson ${ }^{4}$ \\ ${ }^{1}$ Division of Public Health Sciences, Department of Surgery, Washington University in Saint Louis, \\ 660 So. Euclid Avenue, P.O. Box 8100, Saint Louis, MO 63110, USA \\ ${ }^{2}$ Department of Medicine, Washington University in Saint Louis, 4950 Children's place St. Louis, MO 63110, USA \\ ${ }^{3}$ Siteman Cancer Center, Washington University in Saint Louis, 660 So. Euclid Avenue, P.O. Box 8100, Saint Louis, MO 63110, USA \\ ${ }^{4}$ Division of Gastroenterology, Department of Medicine, Washington University in Saint Louis, 660 So. Euclid Avenue, \\ P.O. Box 8124, Saint Louis, MO 63110, USA
}

Correspondence should be addressed to Aimee S. James, jamesai@wudosis.wustl.edu

Received 16 March 2012; Revised 8 June 2012; Accepted 19 June 2012

Academic Editor: Laura Koehly

Copyright (C) 2012 Aimee S. James et al. This is an open access article distributed under the Creative Commons Attribution License, which permits unrestricted use, distribution, and reproduction in any medium, provided the original work is properly cited.

\begin{abstract}
Familial Adenomatous Polyposis (FAP) is a rare autosomal dominantly inherited colorectal cancer syndrome. Individuals with FAP often undergo colectomy and are recommended to follow several surveillance protocols. Biological relatives of persons with FAP may also be at risk and thus should undergo genetic counseling. Screening adherence, genetic testing, and other health behaviors among individuals with FAP and their relatives are not well characterized. We conducted a cross-sectional self-report survey with individuals who have FAP $(n=35)$ and their biological relatives $(n=15)$. Respondents were recruited through a cancer center registry for inherited colon cancers. Most relatives had undergone colon cancer screening; $40 \%$ had undergone genetic testing. One fifth of respondents with FAP had not undergone an upper endoscopy, contrary to usual recommendations. Cigarette smoking rates were above average and were higher among FAP respondents. Use of vitamin supplements was fairly common, more so among those with FAP. Although most people had been screened, there are areas for improvement, notably for upper endoscopy among individuals with FAP and genetic testing among family members. Several other health-risk behaviors and health concerns other than FAP were identified. Further research into factors contributing to screening rates and other health behaviors in this high-risk population is warranted.
\end{abstract}

\section{Introduction}

Colorectal cancer $(\mathrm{CRC})$ is the third leading cause of cancer deaths in the United States [1]. About $30 \%$ of CRC cases are associated with family history or inherited factors and $5 \%$ are attributed to well-identified inherited gene mutations [2]. Familial adenomatous polyposis (FAP) is a highly penetrant autosomal dominant colon cancer syndrome and is the second most common genetic CRC syndrome. The reported incidence of FAP varies from 1 in 6,000 to 1 in 22,000 individuals [3-5]. Individuals who have FAP often develop hundreds to thousands of colorectal adenomatous polyps and have a near $100 \%$ risk of CRC in the absence of clinical intervention. Thus, most patients with FAP undergo either a complete or subtotal colectomy in young adulthood and are recommended to continue surveillance for extracolonic manifestations such as duodenal and periampullary carcinomas and papillary thyroid cancer. Screening and surveillance protocols vary depending on several factors including the severity of the disease and its type (classic or attenuated FAP), previous surgery, and type of surgery [6].

FAP is associated with mutations in the adenomatous polyposis coli (APC) gene, which results in development of numerous polyps. While FAP is inherited as a highly penetrant autosomal dominant syndrome, up to a third of patients do not have a family history of FAP and may represent a new (de novo) mutation [7]. When a familialspecific mutation can be identified in a patient, it allows 
other family members to be tested, often before any clinical manifestations of disease. Unfortunately, not all FAP patients have an APC gene mutation that can be found; individuals with the greatest polyp burden (e.g., more than 100 polyps) are more likely to have an identifiable APC gene mutation [7-9]. As with many other inherited colorectal cancer syndromes, genetic counseling is strongly recommended for patients and their biologic family members. Studies estimate that uptake of genetic testing in patients with FAP varies widely, and up to $40 \%$ of individuals may not be tested $[10,11]$.

Individuals diagnosed with FAP or at risk for FAP, like those affected by other inherited CRC syndromes, are expected to adhere to a number of screening recommendations. Protocols for postsurgical lower endoscopic surveillance depend on the type of surgery performed but include annual endoscopic surveillance of remnant anorectal tissue [6]. Yearly checkups with a physician should be opportunities to screen for other, extraintestinal complications [12]. Because of the increased risk for duodenal and periampullary cancers, screening of the upper gastrointestinal tract through upper endoscopy should occur periodically, depending on the size, histology, and number of polyps.

Endoscopic screening rates may be low, however. Suboptimal rates for CRC screening have been documented in the general population and among patients at high risk due to family history or inherited conditions [13-18]. For example, individuals with or at-risk for Lynch syndrome, another inherited colorectal cancer syndrome, may be nonadherent to screening recommendations [17, 19]. Nonadherence takes the form of both hypovigilance (i.e., underscreening) or hypervigilance (i.e., overscreening), but among people undergoing genetic testing for Lynch syndrome, screening behaviors were associated with knowing one's mutation status. In general, screening frequency decreases after testing mutation negative [18], but nonadherence may be particularly concerning for individuals whose Lynch syndrome genetic testing is inconclusive [20]. However, only a handful of studies have examined screening and surveillance specifically among individuals who have FAP and their family members $[15,21]$. One previous study in the USA reported that slightly more than half of participants who had an FAP diagnosis had recent colorectal screening; rates were lower for at-risk relatives, suggesting underscreening [16].

Individuals with an inherited CRC syndrome and their relatives remain at risk for other cancers, common chronic diseases, and complications from their disease. A number of health behaviors have been associated with increased risk for CRC and cancer in general, such as cigarette smoking and dietary factors and supplements. Screening for other cancers, such as breast and prostate, may also be important for health promotion. These behaviors may be influenced by the reality of living with FAP, increased cancer risk, or associated with other chronic conditions (e.g., diabetes) that themselves increase risk for CRC. Burton and colleagues reported that among individuals at risk for Lynch syndrome, cancer screening rates were similar to those for the general population, but that health-risk behaviors were more common among individuals with CRC than among those who tested negative for the syndrome [19]. The prevalence of these health behaviors (or health-risk) behaviors has not, to our knowledge, been characterized in a published research study of FAP kindreds.

We were interested in ascertaining the needs of individuals with FAP and their relatives in regard to health behavior, disease management, health information, and how needs might be affected by having an inherited CRC syndrome. To build a foundation for future research, we conducted a cross-sectional survey to explore rates of health/health-risk behavior, use of genetic testing, comorbid chronic diseases faced by families, and understanding of genetics and their illness.

\section{Methods}

2.1. Study Overview. This was a single-center cross-sectional survey study of patients with FAP and their biological relatives. Participants were recruited from a cancer-center based registry of patients with known inherited colon cancer syndromes. Respondents completed a survey and were asked to invite family members to complete a survey as well.

2.2. Participants and Recruitment. Eligible participants were 18 years of age and older, able to speak or understand English, and had a diagnosis of FAP or were related to someone with FAP. There were no additional exclusion criteria. All members of the cancer center's inherited colorectal cancer registry who had been diagnosed with FAP (or were a biologic relative) and had previously provided consent to be contacted for future research studies were mailed an invitation letter and information sheet about the study. A follow-up telephone call was made within two weeks of the mailing. The research team attempted telephone contact at least five times, varying day and time, to maximize the opportunities to reach the registrant. The purpose of the telephone call was to prompt survey completion and answer any questions the individual may have had. If registrants had an email address on file with the registry, invitations were sent by electronic mail.

2.3. Procedures. The invitation letter contained instructions and a password for access to the internet version of the survey. Although the survey was designed to be completed via the internet, participants who were reached by telephone and had not yet completed the survey online were offered the opportunity to complete the survey by telephone. If requested, paper versions of the survey were mailed. The survey took about 30 minutes when completed over the telephone. Participants provided verbal consent and did not receive a monetary incentive. Survey responses were not linked to medical information in the registry. Data were collected over a five month period between November 2010 and April 2011. All procedures and materials were approved by the University's Institutional Review Board.

2.4. Measures. Our survey items were selected to address key areas that our multidisciplinary research team thoughts were 
important for health and wellbeing of individuals with FAP and their family members based on a review of the FAP and colon cancer clinical and epidemiological literature. These included comorbid conditions and health status, health behaviors and cancer screening, and several FAP-specific constructs (e.g., knowledge). All items were self-reported. Most questions were the same for patients with FAP and for relatives, but some were adapted per FAP status or were only asked of those who had FAP.

Demographic and Health Status. Demographic factors assessed included age, sex, race/ethnicity, household income, employment status, marital status, and health insurance; these items were measured using standard questions from national health surveys. We also assessed years of education attained. Health status items included comorbid chronic conditions (e.g., diabetes, hypertension, heart disease) and self-rated health. An open-ended question asked respondents to free list their top health concerns.

Health Behavior and Cancer Screening. Respondents were asked about vaccination for pneumonia (lifetime) and influenza (current season), daily consumption of fruits and vegetables per day, current use of nutritional supplements, and cigarette smoking (current and ever). Participants were asked about CRC screening including fecal occult blood testing, sigmoidoscopy, and colonoscopy, using validated questions [22]. For each screening question, participants were provided a description of a screening test and asked if they had ever had it, and, if yes, when they had their most recent test. Using a similar format, women were asked about mammography and cervical screening and men were asked about prostate specific antigen testing.

FAP-Specific Questions. FAP patients self-reported details of their disease and treatment, including surgeries, patientprovider discussion about FAP and risk, genetic counseling, genetic testing, and outcomes of genetic testing. Additionally, participants were asked about their awareness of family members with cancer or FAP, including how many total relatives were known to be affected and their relation. Finally, participants were asked about cancer-related worry and FAP knowledge.

2.5. Analysis. Data analyses were conducted using SPSS software. Descriptive statistics are reported, stratified by FAP status. Due to the small sample size, we did not test for significance. Values were not adjusted for the potential clustering in the data due to having multiple respondents from the same family.

\section{Results}

3.1. Sample Description. We mailed invitation letters to all eligible registry members $(n=66)$. Of those, 40 completed surveys, 11 did not complete the survey, 1 declined participation, and 14 were not reached. We asked respondents to invite additional biological family members (who were at least age
18) to participate; additional 10 respondents were accrued through this strategy. In total, our sample $(N=50)$ consisted of 35 respondents with a known FAP diagnosis, and 15 of their relatives. FAP status of relatives was either negative or unknown. About half of respondents $(n=26)$ completed the survey online, 12 chose telephone interviews, and 12 returned paper surveys. Most respondents (62.0\%) were female and nearly all (94.0\%) self-identified as White. Few demographic differences were evident between respondents with FAP and relatives (see Table 1), but $65.7 \%$ of those with FAP were married or living with a partner, compared to $80 \%$ of relatives.

3.2. Colon and FAP-Related Screening and Surveillance. Most relatives of patients with FAP had undergone a colonoscopy within the past 5 years ( $87.5 \%$ of those aged 50 and over). Relatives said that they had talked with a doctor about FAP ( $80 \%$ said yes) and had a doctor explain their risks due to being in an FAP family $(66.7 \%)$ and their personal risk for CRC $(80.0 \%)$. Forty percent of relatives had undergone genetic testing for FAP.

All respondents who had FAP had undergone a colonoscopy at some point in their lives, $62.9 \%$ of which occurred in the previous five years. Despite strong recommendations for regular upper endoscopic screening, 20\% of respondents with FAP had either never had upper endoscopy or were unsure. Most of the respondents with FAP had undergone genetic testing $(60.0 \%)$ and knew of other family members who had been tested $(65.7 \%)$.

3.3. Knowledge and Attitudes about FAP and Cancer. Perceived risk of $\mathrm{CRC}$ varied greatly amongst relatives, with one-fifth of respondents perceiving very high risk and onefifth perceiving very low risk. While perceived risk varied but was high for many people, colon cancer worry was generally low. Most respondents (60.0\%) said they "rarely" or "never" worried about getting CRC. Further, relatives endorsed positive attitudes about colon cancer prevention and felt they understood the recommendations; with the majority disagreeing with the statements that "there is not much you can do to lower your chances of getting colon cancer" $(73.3 \%$ disagreed) and "there are so many recommendations about preventing cancer, it is hard to know which to follow" $(66.7 \%$ disagreed).

On the other hand, among relatives, there was some misunderstanding about FAP genetics. A majority $(66.7 \%)$ responded that children who do not inherit FAP can still pass it on to their own children, and $40 \%$ were not sure of the likelihood that a child would inherit the disease from a parent with FAP.

3.4. Health and Other Health Behaviors. Table 2 describes health status and health behaviors among respondents. Although most respondents characterized their health as "good" (44.1\% of FAP, $40.0 \%$ of relatives), there were some differences between those with FAP and their relatives. In particular, $35.3 \%$ of patients with FAP self-rated their health as "excellent" or "very good" compared to $46.6 \%$ of relatives. 
TABle 1: Demographic characteristics of the sample $(N=50)$.

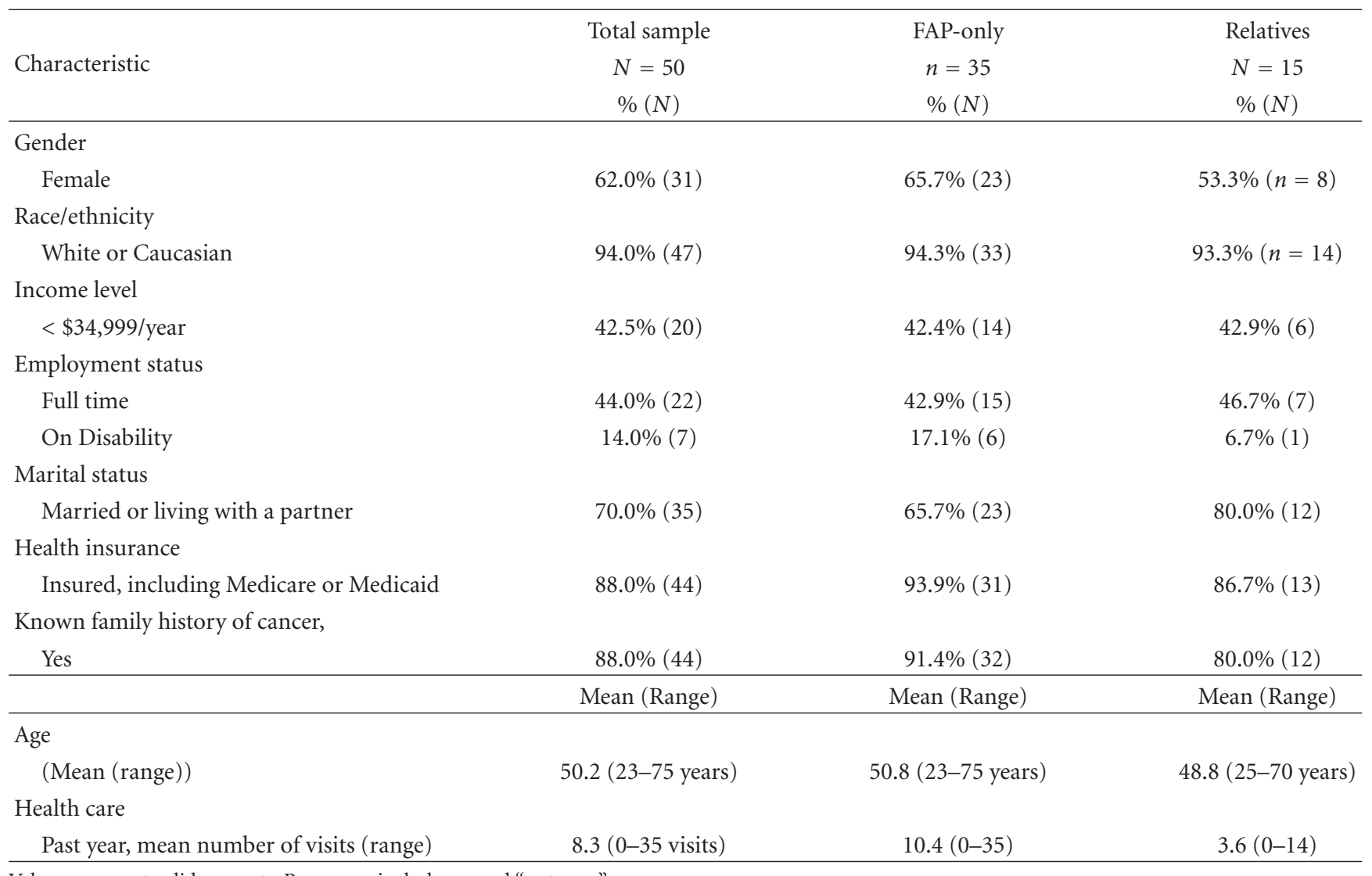

Values represent valid percents. Responses include yes and "not sure."

One-fifth $(20.6 \%)$ of those with FAP rated their health as "fair" or "poor" compared to $13.3 \%$ of relatives.

Preventive care and other screening utilization were consistently high in this sample. Nearly all women age 40 and older had ever had a mammogram (95.7\%), many within the past year $(60.9 \%)$. Cervical cancer screening rates were similarly favorable (96.8\% ever screened; and 80\% screened with the last three years). Mammography and Pap testing rates were similar between FAP and relative respondents. Flu and pneumonia vaccination reports were higher among those with FAP $(64.7 \%$ received the current flu season shot, $28.6 \%$ ever had pneumonia vaccine) than among the relatives (40.0\% flu shot, $20.0 \%$ pneumonia).

Given the shared risk factor profiles of colon cancer and several chronic diseases, we also assessed the presence of other chronic conditions. Half of respondents reported another chronic condition; the overall prevalence did not appear to differ between those with FAP and their relatives. The most commonly reported conditions included high blood pressure (42.9\% of FAP, $46.7 \%$ of relatives), and diabetes $(20.0 \%$ of FAP, and $7.1 \%$ of relatives). Cigarette smoking rates were higher among the respondents with FAP. Among those with FAP, 28.6\% were current smokers, and another $34.3 \%$ had previously smoked but had quit more than 6 months prior. Among relatives, 21.4\% were current smokers, and $35.7 \%$ had quit more than six months prior. Most respondents reported eating two or fewer servings of fruits and two or fewer servings of vegetables each day. Supplement use was higher among those with FAP compared to relatives, including use of Vitamin C (21.9\% of FAP, $6.7 \%$ of relatives), Vitamin E (15.6\% of FAP, $7.1 \%$ of relatives), and Vitamin D (36.4\% of FAP, $3.6 \%$ of relatives). Sixty percent of those with FAP and $40.0 \%$ of relatives reported daily aspirin use. One exception to this trend was calcium supplements, which was higher in relatives (33.3\%) than among those with FAP $(21 \%)$.

\section{Discussion}

This is one of a few studies to examine screening and other health behaviors among individuals with FAP and their biological relatives. FAP is a rare, but well-characterized and prototypical autosomal dominant cancer susceptibility syndrome. The health behaviors of individuals with FAP or their family members have not been well characterized, although it is widely acknowledged that regular screening and surveillance are important for maximizing disease-free survival. Health risk behaviors such as cigarette smoking can detract from overall well being and increase risk for many cancers.

As other studies have found in inherited CRC syndromes [17], many respondents in our sample were vigilant about CRC screening. Screening rates were relatively high in the relatives of FAP patients in this sample-nearly $90 \%$ of those 
TABLe 2: Preventive care and health behaviors of respondents with FAP and their relatives.

\begin{tabular}{|c|c|c|c|}
\hline \multirow{3}{*}{ Characteristic $^{1}$} & Total Sample & FAP-only & Relatives \\
\hline & $N=50$ & $n=35$ & $n=15$ \\
\hline & $\%,(N)$ & $\%(N)$ & $\%(N)$ \\
\hline \multicolumn{4}{|l|}{ Self-rated health } \\
\hline Excellent or Very good & $38.8 \%(19)$ & $35.3 \%(12)$ & $46.6 \%(7)$ \\
\hline Good & $42.9 \%(21)$ & $44.1 \%(15)$ & $40.0 \%(6)$ \\
\hline Fair or Poor & $8.2 \%(4)$ & $20.5 \%(7)$ & $13.3 \%(2)$ \\
\hline \multicolumn{4}{|l|}{ Co-morbidities ${ }^{1}$} \\
\hline Heart disease & $16.7 \%(8)$ & $17.1 \%(6)$ & $15.4 \%(2)$ \\
\hline Diabetes & $16.3 \%(8)$ & $20.0 \%(7)$ & $7.1 \%(1)$ \\
\hline High blood pressure & $44.0 \%(22)$ & $42.9 \%(15)$ & $46.7 \%(7)$ \\
\hline Any comorbidity & $48.0 \%(24)$ & $48.5 \%(17)$ & $46.7 \%(7)$ \\
\hline Cancer, personal history & $28.0 \%(14)$ & $34.3 \%(12)$ & $13.3 \%(2)$ \\
\hline \multicolumn{4}{|l|}{ Vaccination } \\
\hline Flu shot & $57.1 \%(28)$ & $64.7 \%(22)$ & $40.0 \%(6)$ \\
\hline Pneumonia & $28.0 \%(14)$ & $28.6 \%(10)$ & $26.7 \%(4)$ \\
\hline \multicolumn{4}{|l|}{ Mammography ${ }^{2}$} \\
\hline Ever had & $95.7 \%(22)$ & $94.1 \%(16)$ & $100 \%(6)$ \\
\hline Exam within past year & $60.9 \%(14)$ & $58.8 \%(10)$ & $66.7 \%(4)$ \\
\hline \multicolumn{4}{|l|}{ Pap test ${ }^{3}$} \\
\hline Ever had & $96.8 \%(30)$ & $95.7 \%(22)$ & $100 \%(8)$ \\
\hline Exam within three years & $80.6 \%(25)$ & $82.6 \%(19)$ & $75.0 \%(6)$ \\
\hline \multicolumn{4}{|l|}{ PSA test ${ }^{4}$} \\
\hline Ever had & $90.0 \%(9)$ & $100 \%(6)$ & $75.0 \%(3)$ \\
\hline Test within last year & $80.0 \%(8)$ & $83.3 \%(5)$ & $75.0 \%(3)$ \\
\hline \multicolumn{4}{|l|}{ Colonoscopy } \\
\hline Ever had & $94.0 \%(47)$ & $100 \%(35)$ & $80.0 \%(12)$ \\
\hline 50 and over & & $\mathrm{n} / \mathrm{a}$ & $100.0 \%(8)$ \\
\hline Within last 5 years & $66.0 \%(33)$ & $62.9 \%(22)$ & $73.3 \%(11)$ \\
\hline 50 and over & & $\mathrm{n} / \mathrm{a}$ & $87.5 \%(7)$ \\
\hline \multicolumn{4}{|c|}{ Provider recommended CRC Screening } \\
\hline Yes & $70.0 \%(35)$ & $74.3 \%(26)$ & $60.0 \%(9)$ \\
\hline \multicolumn{4}{|l|}{ Upper Endoscopy } \\
\hline Yes, Ever had & $64.0 \%(32)$ & $80.0 \%(28)$ & $26.7 \%(4)$ \\
\hline Not Sure & $6.0 \%(3)$ & $5.7 \%(2)$ & $6.7 \%(1)$ \\
\hline
\end{tabular}

${ }^{1}$ Responses combine yes and "not sure". ${ }^{2}$ Women, age 40 and over (FAP $n=17$; FDR $n=6$ ). ${ }^{3}$ Women, over age 18 (FAP $n=23$, FDR $n=8$ ). ${ }^{4}$ Men, age 50 and over (FAP $n=6$; FDR $n=4)$.

over 50 reported a colonoscopy within the past five years. Although the rate of recent colonoscopy appeared to be lower in FAP patients than relatives, this likely reflects the type of surgery the individual had received and the medical appropriateness of colonoscopy versus other strategies for surveillance of any remaining colon or rectum. We did not have the medical record data to determine what each person's optimal screening interval should be, so some of this may in fact reflect overscreening or misclassification of screening status. However, it should be noted that this rate is higher than has been reported in many studies of FAP kindred $[15,16,21]$. Future studies would benefit from the integration of medical record and patient reported data to better characterize screening adherence.

The relatively high rate of screening may also be reinforced by a high number of unaffected relatives who had discussed their family's history of FAP with a doctor, and the strong support by respondents for the benefits of screening. It may also be influenced by what seemed to be a lack of understanding of the genetics of FAP among relatives, specifically worries that the disease could still be passed down to children even when the parent has tested mutation 
negative. This misconception has also been reported in Lynch syndrome [23]. The high level of interest in genetic testing for CRC [24] and associations between risk perceptions and genetic testing $[25,26]$, when combined with higher rates of cancer screening found in many studies, may reflect a need for reassurance of genetically "unaffected" individuals and a focus on genetic literacy by all health professionals who interact with individuals in families affected by FAP. On the other hand, some researchers have found that screening may decrease (either appropriately or to underscreening) after testing mutation negative for cancer syndromes [18]. Additional studies of genetic literacy [27-29] as well as lay theories of inheritance [30] might shed light on how individuals and families interpret-and cope with-their mutation status.

Other studies have identified higher-risk behaviors among individuals with a familial or genetic risk for CRC [19]. Particularly concerning in our study was the high rate of cigarette smoking (28.6\% FAP and $21.4 \%$ relatives were current smokers). In comparison, 2010 data from the Behavioral Risk Factor Surveillance System for Missouri (our cancer center primarily draws patients from Missouri and neighboring states), indicate that $21 \%$ of adults statewide were current smokers. Respondents in our sample, therefore, smoked at rates equivalent to (for relatives) or higher than (for those with FAP) the statewide average, and much higher than the national average (17.2\% current smokers). This is also equal to or higher than those rates reported for individuals with Lynch syndrome; Burton and colleagues reported that $29.1 \%$ of affected and $12.9 \%$ of unaffected patients were current smokers [19]. Cigarette smoking is a known risk factor for colorectal [31, 32] and other cancers, including those for which FAP also increases risk. Although the relationship between cigarette smoking and malignancy has not yet been investigated in FAP, studies of other inherited CRC syndromes have shown increased risk for polyps [33] or CRC [34] associated with smoking cigarettes. Thus, the seemingly high rate of cigarette smoking is concerning despite our small sample; such findings merit future investigation with larger FAP samples and biologic measurement.

Similarly, other behaviors or conditions that are generally associated with increased cancer risk in the general population, such as low fruit and vegetable consumption, were evident in our sample. Further, our data show that cancer is not the only health concern in this sample: heart disease, hypertension, and diabetes were also reported. In particular, diabetes was reported more often by FAP respondents. Much like the case with cigarette smoking, the potential that diabetes is more common among individuals with FAP may be important because of the increased adenoma $[35,36]$ and CRC risk [37-40] associated with diabetes, even though the link has not been specifically studied in FAP. We were unable to verify disease diagnosis or to explore disease management, or how FAP or a family history of FAP might interact with self-management for other conditions. The health promoting behaviors and concurrent chronic conditions among individuals with inherited CRC syndromes and their biologic relatives may be an important area to study, both in terms of identifying needs and potential interventions, but also examining care coordination and pathways to increased risk.

The data on supplement use in both those diagnosed with FAP and their biological relatives are novel and interesting. There is evidence for chemopreventive agents for CRC in average-risk and FAP patients [41]. There has been at least one published study of aspirin use in FAP patients demonstrating a reduction in polyp burden [42], and several good-quality studies have found a reduction in risk for recurrence of polyps, and the incidence of advanced adenomas and CRC with aspirin use in general [43-45]. In our sample, reported daily aspirin use was higher for FAP than non-FAP respondents, while both rates $(60 \%$ and $40 \%$ ) were fairly high. Other vitamin supplementation was relatively common $(>15 \%$ of respondents with FAP answering yes). As far as we know, there are no studies of the impact of Vitamins C, E, and D in individuals with FAP [46], though some studies mention vitamin deficiencies [47] and avoidance of certain foods [48] after colectomy. So far, data on Vitamin D and calcium supplementation in the reduction of adenoma occurrence or the prevention of CRC has been suggestive but not conclusive [49]. The dosage, or whether supplementation was discussed with a clinical provider, is not evident from our data. However, the extent of supplement use, the reasons why patients seek out supplements, and the potential clinical implications warrant further study.

Our study is subject to some limitations. Respondents were drawn from a registry at the cancer center or were a biological relative of a registrant, and thus they may have more interaction with, or access to, specialists in FAP than would other FAP families. We were unable to link medical records to survey answers or verify self-reported diagnoses. Additionally, if our participants were seeing providers more often, there may be detection bias in their self-reported chronic conditions. The relatives in our study may also represent those family members closest to the person with FAP, or who were most receptive to health screening and discussion. This weakness is inherent in studies of kindreds affected by inherited syndromes, which primarily recruit clinically unaffected relatives through the patient. Thus, our rates of screening are probably higher than what we would find in a community-based sample of FAP kindred. Indeed, as we already stated, studies have reported higher rates of screening among individuals who are associated with polyposis or other inherited cancer registries. Although we cannot determine optimal screening intervals or strategies for respondents with FAP in our sample and our estimates for colorectal screening and upper endoscopy adherence are not tailored to the individual's risk, it is worth noting that all the participants who are in the registry are offered protocolbased screening (periodic upper and lower endoscopy). Our findings regarding chronic disease may be related to a detection bias if the patients saw a healthcare provider more often. Lastly, our sample size precluded advanced statistical testing, our descriptive analysis did not account for family-level variance or correlation, and we did not ask the relationship between patients and relatives. 


\section{Conclusions}

Our findings in individuals with FAP and their biological relatives revealed relatively high rates of endoscopic screening, but some gaps in surveillance and screening, particularly with recommendations for upper endoscopy. There were also indications of lack of knowledge about FAP and its genetics and inheritance patterns.

These issues are relevant for other populations at highrisk because of genetic syndromes. There were some similarities between our health risk data and that reported elsewhere for other CRC syndromes. Clearly, patients and families affected by inherited syndromes are concurrently dealing with other chronic conditions (in this sample, most notably heart disease and diabetes), which may complicate their health-maintenance perceptions and behaviors. Additionally, much like other studies, we found a range of reactions among relatives including continued concern about FAP or its transmission. Patient-provider discussions about the genomics of disease could be targeted to address some of these concerns, and also widened to address other chronic conditions that might affect how individuals deal with their cancer syndrome.

Therefore, it is critical to characterize the prevalence and predictors of health and health behaviors among FAP kindred, and to explore how the increased risk associated with this syndrome may affect other behaviors and conditions. Additionally, future studies may try to extend the study sample beyond a cancer center or registry, in order to better capture the experience of FAP in the general population, and to combine patient-reported outcomes with objectively collected data.

\section{Acknowledgments}

A portion of Dr. James' time was supported by funding by the Barnes-Jewish Hospital Foundation. Dr. Davidson was supported in part by Grants (HL-38180, DK-56260, and DDRCC DK-52574) and through funds from the Buehrle Family Foundation. Other support for this study was provided by Siteman Cancer Center. The authors sincerely thank our participants for making this study possible.

\section{References}

[1] ACS, Cancer Facts and Figures, 2011, American Cancer Society, Atlanta, Ga, USA, 2011.

[2] P. Lichtenstein, N. V. Holm, P. K. Verkasalo et al., "Environmental and heritable factors in the causation of cancer: analyses of cohorts of twins from Sweden, Denmark, and Finland," The New England Journal of Medicine, vol. 343, no. 2, pp. 78-85, 2000.

[3] S. Bülow, T. Faurschou Nielsen, C. Bülow, M. L. Bisgaard, L. Karlsen, and F. Moesgaard, "The incidence rate of familial adenomatous polyposis. Results from the Danish Polyposis Register," International Journal of Colorectal Disease, vol. 11, no. 2, pp. 88-91, 1996.

[4] E. Half, D. Bercovich, and P. Rozen, "Familial adenomatous polyposis," Orphanet Journal of Rare Diseases, vol. 4, no. 1, article 22, 2009.
[5] K. W. Jasperson, T. M. Tuohy, D. W. Neklason, and R. W. Burt, "Hereditary and familial colon cancer," Gastroenterology, vol. 138, no. 6, pp. 2044-2058, 2010.

[6] P. M. Lynch, "Prevention of colorectal cancer in high-risk populations: the increasing role for endoscopy and chemoprevention in FAP and HNPCC," Digestion, vol. 76, no. 1, pp. 6876, 2007.

[7] L. L. Strate and S. Syngal, "Hereditary colorectal cancer syndromes," Cancer Causes and Control, vol. 16, no. 3, pp. 201213, 2005.

[8] O. M. Sieber, H. Lamlum, M. D. Crabtree et al., "Whole-gene APC deletions cause classical familial adenomatous polyposis, but not attenuated polyposis or "multiple" colorectal adenomas," Proceedings of the National Academy of Sciences of the United States of America, vol. 99, no. 5, pp. 2954-2958, 2002.

[9] S. Aretz, D. Stienen, S. Uhlhaas et al., "Large submicroscopic genomic APC deletions are a common cause of typical familial adenomatous polyposis," Journal of Medical Genetics, vol. 42, no. 2, pp. 185-192, 2005.

[10] K. F. L. Douma, N. K. Aaronson, H. F. A. Vasen, and E. M. A. Bleiker, "Psychosocial issues in genetic testing for familial adenomatous polyposis: a review of the literature," PsychoOncology, vol. 17, no. 8, pp. 737-745, 2008.

[11] K. F. L. Douma, E. M. Bleiker, N. K. Aaronson et al., "Distress and use of professional psychosocial support in individuals from families at high-risk for familial adenomatous polyposis (FAP)," Psycho-Oncology, vol. 17, pp. S159-S159, 2008.

[12] J. Church and C. Simmang, "Practice parameters for the treatment of patients with dominantly inherited colorectal cancer (familial adenomatous polyposis and hereditary nonpolyposis colorectal cancer)," Diseases of the Colon and Rectum, vol. 46, no. 8, pp. 1001-1012, 2003.

[13] CDC, "Cancer screening-United States, 2010," Morbidity and Mortality Weekly Report, vol. 61, no. 3, pp. 41-45, 2012.

[14] E. K. L. Mallinson, K. F. Newton, J. Bowen et al., "The impact of screening and genetic registration on mortality and colorectal cancer incidence in familial adenomatous polyposis," Gut, vol. 59, no. 10, pp. 1378-1382, 2010.

[15] S. Michie, V. Collins, D. Eccles et al., "Likelihood of attending bowel screening after a negative genetic test result: the possible influence of health professionals," Genetic Testing, vol. 6, no. 4, pp. 307-311, 2002.

[16] A. Y. Kinney, B. Hicken, S. E. Simonsen et al., "Colorectal cancer surveillance behaviors among members of typical and attenuated FAP families," American Journal of Gastroenterology, vol. 102, no. 1, pp. 153-162, 2007.

[17] E. M. Stoffel, R. C. Mercado, W. Kohlmann et al., "Prevalence and predictors of appropriate colorectal cancer surveillance in lynch syndrome," American Journal of Gastroenterology, vol. 105, no. 8, pp. 1851-1860, 2010.

[18] D. W. Hadley, J. F. Jenkins, E. Dimond, M. De Carvalho, I. Kirsch, and C. G. S. Palmer, "Colon cancer screening practices after genetic counseling and testing for hereditary nonpolyposis colorectal cancer," Journal of Clinical Oncology, vol. 22, no. 1, pp. 39-44, 2004.

[19] A. M. Burton, S. K. Peterson, S. K. Marani et al., "Health and lifestyle behaviors among persons at risk of Lynch syndrome," Cancer Causes and Control, vol. 21, no. 4, pp. 513-521, 2010.

[20] A. L. Ersig, D. W. Hadley, and L. M. Koehly, "Colon cancer screening practices and disclosure after receipt of positive or inconclusive genetic test results for hereditary nonpolyposis colorectal cancer," Cancer, vol. 115, no. 18, pp. 4071-4079, 2009. 
[21] K. F. L. Douma, E. M. A. Bleiker, N. K. Aaronson et al., "Longterm compliance with endoscopic surveillance for familial adenomatous polyposis," Colorectal Disease, vol. 12, no. 12, pp. 1198-1207, 2010.

[22] S. W. Vernon, H. Meissner, C. Klabunde et al., "Measures for ascertaining use of colorectal cancer screening in behavioral, health services, and epidemiologic research," Cancer Epidemiology Biomarkers and Prevention, vol. 13, no. 6, pp. 898-905, 2004.

[23] K. Bartuma, M. Nilbert, and C. Carlsson, "Family perspectives in lynch syndrome becoming a family at risk, patterns of communication and influence on relations," Hereditary Cancer in Clinical Practice, vol. 10, no. 1, article 6, 2012.

[24] K. Glanz, J. Grove, C. Lerman, C. Gotay, and L. Le Marchand, "Correlates of intentions to obtain genetic counseling and colorectal cancer gene testing among at-risk relatives from three ethnic groups," Cancer Epidemiology Biomarkers and Prevention, vol. 8, no. 4, part 2, pp. 329-336, 1999.

[25] A. M. Codori, G. M. Petersen, D. L. Miglioretti et al., "Attitudes toward colon cancer gene testing: factors predicting test uptake," Cancer Epidemiology Biomarkers and Prevention, vol. 8, no. 4, part 2, pp. 345-351, 1999.

[26] G. M. Petersen, E. Larkin, A. M. Codori et al., "Attitudes toward colon cancer gene testing: survey of relatives of colon cancer patients," Cancer Epidemiology Biomarkers and Prevention, vol. 8, no. 4, part 2, pp. 337-344, 1999.

[27] C. M. Condit, "Public attitudes and beliefs about genetics," Annual Review of Genomics and Human Genetics, vol. 11, pp. 339-359, 2010.

[28] C. M. McBride, D. Bowen, L. C. Brody et al., "Future health applications of genomics: priorities for communication, behavioral, and social sciences research," American Journal of Preventive Medicine, vol. 38, no. 5, pp. 556-565, 2010.

[29] C. M. Condit, "Public understandings of genetics and health," Clinical Genetics, vol. 77, no. 1, pp. 1-9, 2010.

[30] M. McAllister, "Personal theories of inheritance, coping strategies, risk perception and engagement in hereditary nonpolyposis colon cancer families offered genetic testing," Clinical Genetics, vol. 64, no. 3, pp. 179-189, 2003.

[31] K. K. Tsoi, C. Y. Y. Pau, W. K. K. Wu et al., "Cigarette smoking and the risk of colorectal cancer: a meta-analysis of prospective cohort studies," Clinical Gastroenterology and Hepatology, vol. 7, no. 6, pp. 682.e5-688.e5, 2009.

[32] P. J. Limburg, R. A. Vierkant, J. R. Cerhan et al., "Cigarette smoking and colorectal cancer: long-term, subsite-specific risks in a cohort study of postmenopausal women," Clinical Gastroenterology and Hepatology, vol. 1, no. 3, pp. 202-210, 2003.

[33] R. M. Winkels, A. Botma, F. J. B. van Duijnhoven et al., "Smoking increases the risk for colorectal adenomas in patients with Lynch syndrome," Gastroenterology, vol. 142, no. 2, pp. 241-247, 2012.

[34] P. Watson, R. Ashwathnarayan, H. T. Lynch, and H. K. Roy, "Tobacco use and increased colorectal cancer risk in patients with hereditary nonpolyposis colorectal cancer (Lynch syndrome)," Archives of Internal Medicine, vol. 164, no. 22, pp. 2429-2431, 2004.

[35] R. Eddi, A. Karki, A. Shah et al., "Association of type 2 diabetes and colon adenomas," Journal of Gastrointestinal Cancer, vol. 43, no. 1, pp. 87-92, 2012.

[36] T. Morita, S. Tabata, M. Mineshita, T. Mizoue, M. A. Moore, and S. Kono, "The metabolic syndrome is associated with increased risk of colorectal adenoma development: the SelfDefense Forces health study," Asian Pacific Journal of Cancer Prevention, vol. 6, no. 4, pp. 485-489, 2005.

[37] J. Luo, R. Chlebowski, J. Wactawski-Wende et al., "Diabetes mellitus as a risk factor for gastrointestinal cancers among postmenopausalwomen," Cancer Causes and Control. In press.

[38] L. Deng, Z. Gui, L. Zhao et al., "Diabetes mellitus and the incidence of colorectal cancer: an updated systematic review and meta-analysis," Digestive Diseases and Sciences, vol. 57, no. 6, pp. 1576-1585, 2012.

[39] J. He, D. O. Stram, L. N. Kolonel, B. E. Henderson, L. Le Marchand, and C. A. Haiman, "The association of diabetes with colorectal cancer risk: the Multiethnic Cohort," British Journal of Cancer, vol. 103, no. 1, pp. 120-126, 2010.

[40] S. C. Larsson, N. Orsini, and A. Wolk, "Diabetes mellitus and risk of colorectal cancer: a meta-analysis," Journal of the National Cancer Institute, vol. 97, no. 22, pp. 1679-1687, 2005.

[41] P. A. Jänne and R. J. Mayer, "Chemoprevention of colorectal cancer," The New England Journal of Medicine, vol. 342, no. 26, pp. 1960-1968, 2000.

[42] J. Burn, D. T. Bishop, P. D. Chapman et al., "A randomized placebo-controlled prevention trial of aspirin and/or resistant starch in young people with familial adenomatous polyposis," Cancer Prevention Research, vol. 4, no. 5, pp. 655-665, 2011.

[43] J. A. Baron, "Epidemiology of non-steroidal anti-inflammatory drugs and cancer," Progress in Experimental Tumor Research, vol. 37, pp. 1-24, 2003.

[44] E. J. Jacobs, M. J. Thun, E. B. Bain, C. Rodriguez, S. J. Henley, and E. E. Calle, "A large cohort study of long-term daily use of adult-strength aspirin and cancer incidence," Journal of the National Cancer Institute, vol. 99, no. 8, pp. 608-615, 2007.

[45] E. Flossmann and P. M. Rothwell, "Effect of aspirin on long-term risk of colorectal cancer: consistent evidence from randomised and observational studies," The Lancet, vol. 369, no. 9573, pp. 1603-1613, 2007.

[46] K. Cooper, H. Squires, C. Carroll et al., "Chemoprevention of colorectal cancer: systematic review and economic evaluation," Health Technology Assessment, vol. 14, no. 32, pp. 1-206, 2010.

[47] S. A. Buckman and C. P. Heise, "Nutrition considerations surrounding restorative proctocolectomy," Nutrition in Clinical Practice, vol. 25, no. 3, pp. 250-256, 2010.

[48] K. Almendingen, O. Fausa, A. T. Høstmark et al., "Serum nutrients and habitual dietary intake in colectomized FAP patients in Norway," European Journal of Nutrition, vol. 48, no. 3, pp. 129-136, 2009.

[49] E. Half and N. Arber, "Colon cancer: preventive agents and the present status of chemoprevention," Expert Opinion on Pharmacotherapy, vol. 10, no. 2, pp. 211-219, 2009. 


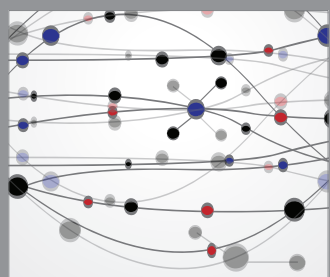

The Scientific World Journal
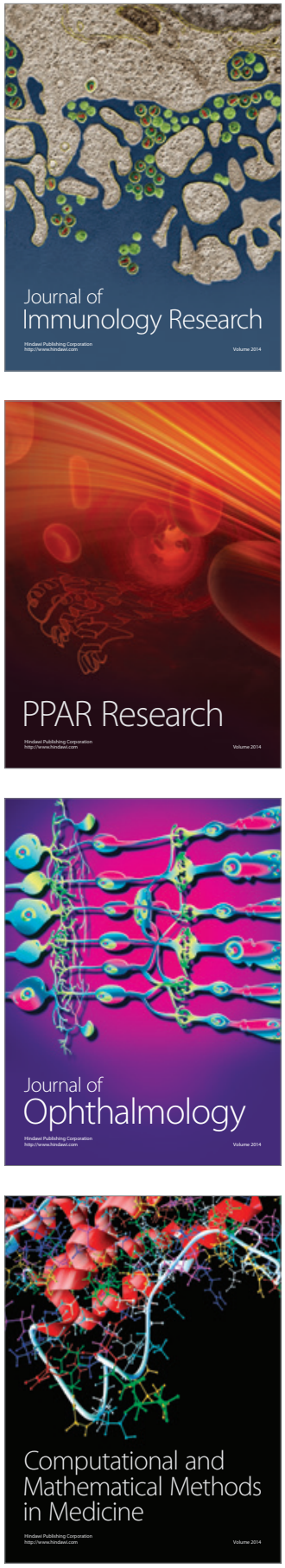

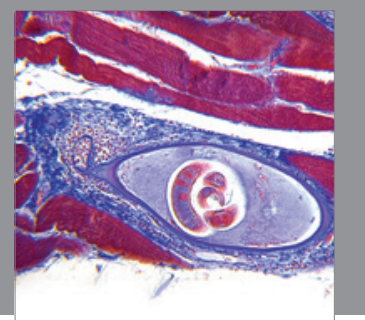

Gastroenterology

Research and Practice
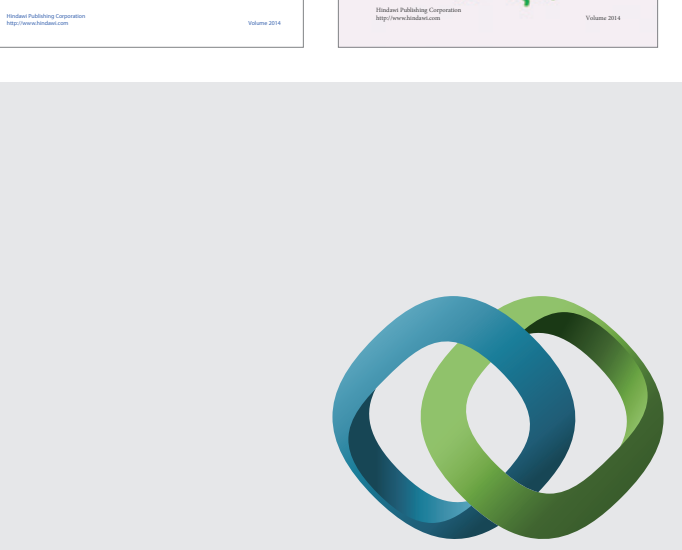

\section{Hindawi}

Submit your manuscripts at

http://www.hindawi.com
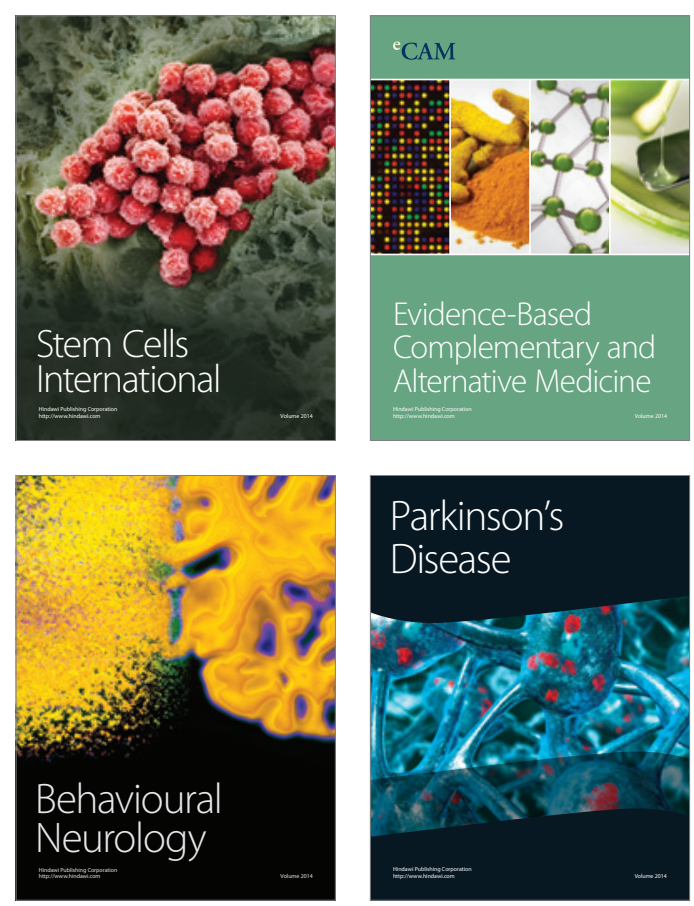

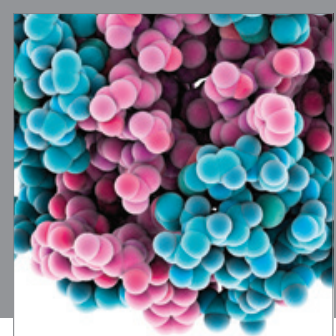

Journal of
Diabetes Research

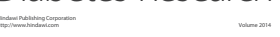

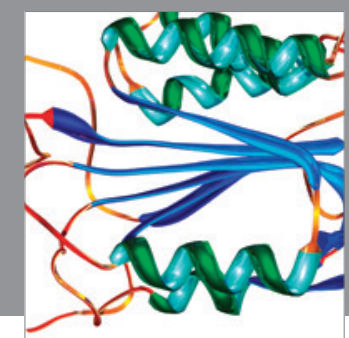

Disease Markers
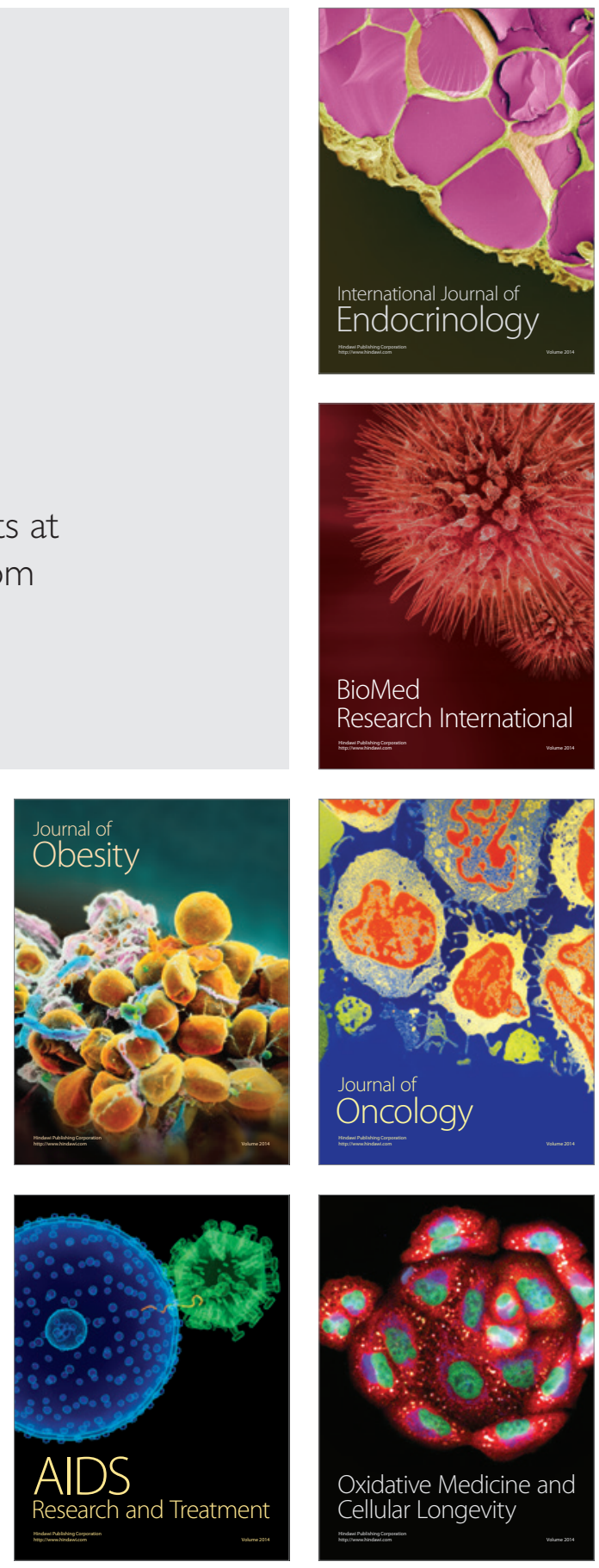